\title{
AVALIAÇÃO DO EFEITO DE FOSFITOS NA REDUÇÃO DA VARÍOLA (ASPERISPORIUM CARICAE) DO MAMOEIRO (CARICA PAPAYA $)^{1}$
}

\author{
ALEXEI DE CAMPOS DIANESE ${ }^{2}$, LUIZ EDUARDO BASSAY BLUM $^{3}$, JAQUELINE BARBOSADUTRA $^{4}$, \\ LEONARDO FERREIRALOPES ${ }^{4}$, MARIANACOELHO SENA $^{4}$, LEANDRO FERREIRADE FREITAS $^{4}$
}

RESUMO - O controle da varíola do mamoeiro é necessário; portanto, propôs-se, neste estudo, avaliar o efeito de aplicações foliares de fosfitos (K, Ca, $\mathrm{Mg}$ e $\mathrm{Cu}$ ) sobre a doença. Dois fosfitos e o fungicida Fosetyl-Al foram testados em condições de campo, com plantas naturalmente infectadas pelo patógeno. Dez fosfitos e o fungicida Fosetyl-Al foram testados sob telado também com plantas naturalmente infectadas por A. caricae. Tanto no experimento de campo quanto sob telado, os tratamentos contendo fosfito reduziram a severidade da doença.

Termos para indexação: fosfito, varíola, Asperisporium caricae, controle, mamoeiro

\section{EVALUATION OF PHOSPHITE APPLICATIONS FOR SEVERITY OF PAPAYA BLACK SPOT (ASPERISPORIUM CARICAE)}

\begin{abstract}
The control of black spot of papaya is necessary, therefore, the objective of this study was to evaluate the effect of foliar applications of phosphites $(\mathrm{K}, \mathrm{Ca}, \mathrm{Mg}$ and $\mathrm{Cu})$ on papaya black spot. Two phosphites and the fungicide Fosetyl-Al were tested on a field trial with papaya plants naturally infected by the pathogen. Ten phosphites and the fungicide Fosetyl-Al were tested in a greenhouse trial, also with plants naturally infected by A. caricae. In both, field and greenhouse trials, the phosphite treatments reduced black spot severity.
\end{abstract}

Index terms: phosphite, black spot, Asperisporium caricae, control, papaya.

O mamoeiro (Carica papaya L.) é originário da América do Sul e é cultivado principalmente em países de clima tropical (Boteon, 2005). O Brasil é um dos líderes mundiais na produção dessa fruta, e a Bahia e o Espírito Santo são os principais produtores nacionais (Brapex, 2007; Boteon, 2005). A varíola [Asperisporium caricae (Speg.) Maubl.] ou pinta-preta é uma das doenças mais comuns do mamoeiro, incitando manchas em folhas e frutos (Resende \& Fancelli, 1997; Ventura et al., 2003). As lesões nos frutos afetam a aparência, depreciando o valor de mercado, além de facilitar a infecção por patógenos pós-colheita (Ventura et al., 2003).

A severidade da pinta-preta está diretamente relacionada com o período de chuvas, época em que se deve iniciar o controle da doença (Zambolim et al., 2006). A aplicação de fungicidas ocorre de forma preventiva ou ao surgimento dos primeiro sintomas (Santana et al., 2007). Produtos à base de chlorotalonil + tiophanato metílico, tebuconazole, azoxystrobin e famoxadone + mancozeb demonstraram boa eficiência no controle da varíola (Celoto et al., 2003; Santana, 2004; Tatagiba \& Aguilar, 2005).

Um produto utilizado no manejo de doenças de plantas, inclusive em espécies arbóreas, é o fosfito de potássio, sendo indicado para o controle de oomicetos (Pythium Pringsh e Phytophthora de Bary) e de fungos causadores de podridões em hastes e frutos (McDonald et al., 2001; Holderness, 1992; Smillie, 1989; Panicker \& Gangadharan, 1999; Hagan, 2005).

Os estudos sobre a utilização de fosfitos para o controle de doenças causadas por patógenos não-pertencentes ao grupo dos oomicetos são recentes, como, por exemplo, o trabalho de Brackmann et al. (2004) em frutos de maçã (Malus domestica Borkh.) para o controle de Penicillium Link e de Ribeiro Júnior et al. (2006) para o controle de Verticillium dahliae Kleb. em cacaueiro.

Portanto, o objetivo deste estudo foi avaliar o efeito da aplicação foliar de fosfitos sobre a ocorrência de varíola em plantas de mamoeiro sob condições de campo e telado, e, dessa forma, incrementar o uso de fosfitos como mais uma ferramenta no manejo integrado de patógenos.

O experimento de campo foi conduzido de dezembro de 2004 a julho de 2005, na Estação Experimental de Biologia da Universidade de Brasília (Brasília-DF, Brasil), usando diversos genótipos de mamão: 'NT Red' 'Baixinho de St. Amália', 'Golden', 'Grampola' e 'Sunrise Solo' do grupo Solo e 'Cross Paris', 'Tailândia Verde', 'Tailândia Roxo' e 'Tailândia Roxão' do grupo Formosa (Tabela 1). Aárea do experimento, de aproximadamente $1.500 \mathrm{~m}^{2}$, tem histórico de elevada severidade de varíola nas plantas (Dianese et al., 2007).

${ }^{1}$ (Trabalho 202-07). Recebido em: 20-08-2007. Aceito para publicação em: 06-06-2008.

${ }^{2}$ Pesquisador A, parte da tese de doutorado, CPAC - Embrapa Cerrados, Planaltina, DF, 73310-970, alexei.dianese@cpac.embrapa.br; apoio parcial da CAPES e do CNPq.

${ }^{3}$ Professor, UnB, Dep. Fitopatologia, Brasília, DF, 70910-900, luizblum@unb.br, Bolsista de Produtividade / CNPq.

${ }^{4}$ Ex-alunos, bolsistas de Iniciação Científica / CNPq, Faculdade de Agronomia e Veterinária, Brasília, DF, 70910-900, jackedutra@gmail.com, leolopes21@gmail.com, marianasena@agricultura.gov.br, leandroffreitas@yahoo.com.br. 
A preparação da área iniciou-se em agosto de 2004. O espaçamento foi de $2,5 \mathrm{~m}$ entre linhas e $2,2 \mathrm{~m}$ entre covas $(40 \mathrm{~cm}$ x $40 \mathrm{~cm}$ x $40 \mathrm{~cm})$. Nas covas, foi aplicado calcário dolomítico $(68,5$ $\%$ PRNT) e incorporado ao solo em uma taxa de $200 \mathrm{~g}$. Três semanas depois, cada cova recebeu $125 \mathrm{~g}$ de $4-16-8$ (N-P-K) e $5 \mathrm{~g}$ de borax-10. Após sete dias, ocorreu o plantio das mudas. Em seguida, cada cova recebeu mensalmente $125 \mathrm{~g}$ de 4-16-8 (N-PK) depositados na superfície do solo. Na irrigação das linhas, foi utilizada fita santeno tipo I com microperfurações a cada $15 \mathrm{~cm}$ e pressão máxima de $0,80 \mathrm{~kg} \mathrm{~cm}^{-2}$ (Santeno Sistemas de Irrigação).

Sementes das variedades dos grupos Solo e Formosa foram semeadas em bandejas de 72 células contendo o substrato 'Plantmax' (casca de pínus, vermiculita e turfa - Eucatex Agro) e 'Osmocote' (14-14-14 - N-P-K - Scotts Co.) na concentração de 5 $\mathrm{g} \mathrm{L}^{-1}$ de Plantmax, e mantidas em área protegida por telado. Duas mudas foram plantadas por cova, separadas pela distância de 30 $\mathrm{cm}$.

O desenho experimental foi o de blocos ao acaso. Duas linhas consecutivas foram plantadas com cada cultivar. $\mathrm{O}$ experimento teve quatro tratamentos: Fosfito $\left(\mathrm{P}_{2} \mathrm{O}_{5} 40 \%+\mathrm{K}\right.$ $\left.20 \%, 150 \mathrm{~mL} \mathrm{hL}^{-1}\right)$; Fosfito $\left(\mathrm{P}_{2} \mathrm{O}_{5} 40 \%+\mathrm{K} 20 \%, 250 \mathrm{~mL} \mathrm{hL}^{-1}\right)$; Fosetyl-Al 80 \% (250 g), e Controle (Testemunha sem aplicação de produtos). Em cada cultivar, quatro plantas foram pulverizadas por tratamento, duas em cada fileira. Cada repetição dos tratamentos consistiu em uma planta por cultivar. As aplicações semanais foram feitas usando um pulverizador manual costal (20 L). Os produtos foram aplicados sobre frutos e folhas até o ponto de escorrimento. As aplicações começaram quando apareceram os primeiros sintomas de varíola em dezembro/2004 e estenderamse até a última semana de junho/2005 (26 aplicações no total). As avaliações semanais foram feitas levando em consideração folhas e frutos separadamente, usando a seguinte escala (Agrios, 1997): $1=$ de 0 a $3 \%$ da superfície de folhas/frutos coberta por lesões; $2=$ de 4 a $6 \%$ da superfície de folhas/frutos coberta por lesões; $3=$ de 7 a $14 \%$ da superfície de folhas/frutos coberta por lesões; $4=$ de 15 a $24 \%$ da superfície de folhas/frutos coberta por lesões; $5=$ de 25 a $50 \%$ da superfície de folhas/frutos coberta por lesões; $6=$ acima de $50 \%$ da superfície de folhas/frutos coberta por lesões.

Os dados foram submetidos à análise de variância de dupla entrada, com repetição de medidas no tempo. Se significativas, as médias dos tratamentos em cada genótipo foram comparadas pelo teste de Tukey $(\mathrm{P}=5 \%$ ) (Tabelas $1 \mathrm{e} 2)$. Esses testes foram executados usando o software SigmaStat 2,0 (Jandel Corporation, 1995).

No experimento sob telado, as sementes da cultivar 'Sunrise Solo' foram semeadas em bandejas de 72 células, contendo substrato 'Plantmax' (Eucatex Agro) e $5 \mathrm{~g} \mathrm{~L}^{-1}$ de solo de $14(\mathrm{~N})-14(\mathrm{P})-14(\mathrm{~K})$ (Osmocote - Scotts Co.), em novembro de 2004. Ao atingirem cerca de $10 \mathrm{~cm}$ de altura ( 15 de dezembro de 2004), as mudas foram transplantadas para vasos de 1,5 L contendo Latossolo Vermelho com textura média, previamente tratado com brometo de metila. Os vasos eram irrigados, quando necessário, utilizando-se de uma mangueira de jardim. A temperatura sob o telado foi de $24^{\circ} \mathrm{C}+/-4^{\circ} \mathrm{C}$

As pulverizações iniciaram-se em março de 2005, quando apareceram os primeiros sintomas nas folhas, e foram interrompidas no início de abril, devido à falta de sintomas. Após cerca de quarenta e cinco dias, os produtos voltaram a ser pulverizados até a segunda quinzena de junho, quando o experimento terminou devido às condições climáticas adversas (temperatura acima de $28^{\circ} \mathrm{C}$ e umidade relativa do ar abaixo de 20\%). Foram onze tratamentos (Tabelas 3 e 4) com aplicações foliares semanais, utilizando um pulverizador manual de $500 \mathrm{~mL}$. Os produtos foram aplicados até o ponto de escorrimento.

O delineamento experimental foi o de blocos ao acaso, com cinco repetições. Cada repetição continha cinco vasos, cada qual com uma muda. A severidade da varíola nas folhas foi avaliada em cada planta, utilizando-se a escala diagramática descrita anteriormente. A análise de variância e comparação entre médias, de cada tratamento, foi realizada através do programa SigmaStat 2.0 da Jandel Corporation (copyright @ 1995).

No experimento de campo, embora as avaliações tenham começado em dezembro/2004, somente a partir de abril/2005 que diferenças significativas entre os tratamentos foram verificadas. Os três tratamentos químicos mantiveram os níveis de severidade da varíola nos frutos significativamente mais baixos do que no controle (sem a aplicação de qualquer produto) na grande maioria das cultivares (Tabela 1). No entanto, houve certa irregularidade no desempenho dos tratamentos em alguns dos genótipos ('Grampola', 'Sunrise Solo', 'Tailândia Verde'e 'Tailândia Roxão'), provavelmente devido a diferenças em termos de suscetibilidade à varíola (Dianese et al., 2007) (Tabela 1). Os dois fosfitos e o tratamento contendo o fungicida fosetyl-Al também diferiram significativamente do controle, na maioria das avaliações de severidade nas folhas (Tabela 2). Além disso, não houve diferença significativa entre os tratamentos químicos na média final de severidade da doença; contudo, essa média foi significativamente mais baixa do que no controle (sem aplicação de qualquer produto) (Tabelas 1 e 2). Os sintomas de varíola foram mais severos na folhagem do que nos frutos, tanto que, no genótipo 'Tailândia Roxo', houve diminuição significativa dos sintomas nos frutos dos mamoeiros que receberam os tratamentos, mas o mesmo não ocorreu na folhagem, provavelmente devido à alta suscetibilidade das folhas desse genótipo à varíola (Tabelas 1 e 2).

No experimento sob telado, todos os tratamentos apresentaram valores significativamente inferiores $(\sim 1,8-2,9)$ ao controle $(\sim 3,5)$, no primeiro ensaio, sendo que o tratamento $\mathrm{P}_{2} \mathrm{O}_{5}$ $25 \%+\mathrm{Cu} 5 \%(\sim 1,8)$ foi o mais efetivo (Tabela 3$)$. No entanto, os tratamentos $\mathrm{P}_{2} \mathrm{O}_{5} 30 \%+\mathrm{Ca} 7 \%, \mathrm{P}_{2} \mathrm{O}_{5} 30 \%+\mathrm{Mg} 4 \%$ e $\mathrm{P}_{2} \mathrm{O}_{5} 25 \%$ $+\mathrm{Cu} 5 \%$ provocaram fitotoxidez (Tabela 3 ) nas folhas dos mamoeiros (“Sunrise Solo') e, por isso, não foram utilizados na repetição do experimento (Tabela 4). No segundo ensaio, notouse diminuição $(\sim 2,5-2,9)$ na severidade da varíola, com o controle apresentando valor médio de severidade da doença em torno de 3,1 (Tabela 4). Ainda assim, a maioria dos tratamentos apresentou valor médio de severidade significativamente inferior ao controle, sendo que os tratamentos $\mathrm{P}_{2} \mathrm{O}_{5} 40 \%+\mathrm{K} 20 \%\left(250 \mathrm{~mL} \mathrm{hL}^{-1}\right)$ e $\mathrm{P}_{2} \mathrm{O}_{5}$ $40 \%+\mathrm{Mg} 6 \%\left(150 \mathrm{~mL} \mathrm{hL}^{-1}\right)$ obtiveram as menores médias $(\sim 2,5)$ (Tabela 4). A doença foi menos severa sob condições de telado do que no campo, o que pode ser comprovado ao se compararem as médias de severidade dos controles nas duas áreas (Tabelas 
$1 ; 2 ; 3$ e 4$)$

Concluindo, a varíola é uma das principais doenças da cultura do mamoeiro (Ventura et al., 2003). No entanto, só recentemente, iniciaram-se estudos epidemiológicos sobre a doença, e começaram a ser testadas estratégias de controle com diferentes fungicidas (Santos \& Barreto, 2003; Celoto et al., 2003; Santana, 2004; Tatagiba \& Aguilar, 2005). No presente estudo, embora todos os produtos testados (campo e telado) tenham reduzido a severidade de varíola, significativamente, quando comparados ao controle, tanto as folhas quanto os frutos apresentaram sintomas típicos da doença. A tolerância ao dano causado por doenças em frutos destinados ao mercado internacional é extremamente baixa, e, às vezes, uma única lesão é o bastante para rejeitar um lote. Logo, o uso de fosfitos pode ser encarado como uma ferramenta a mais, que, em conjunto com os fungicidas tradicionais e algumas outras técnicas, auxiliariam no desenvolvimento de estratégias de manejo eficaz para a varíola do mamoeiro.

TABELA 1 - Efeito de fosfitos na severidade da varíola (Asperisporium caricae) em frutos de nove genótipos de mamoeiro, entre abril e julho de 2005, em condições de campo (Brasília-DF).

\begin{tabular}{|c|c|c|c|c|c|c|c|c|c|c|}
\hline \multirow[b]{2}{*}{ Tratamento } & \multicolumn{10}{|c|}{ Genótipo - Severidade $(1-6)$ de varíola } \\
\hline & $1^{(1)}$ & 2 & 3 & 4 & 5 & 6 & 7 & 8 & 9 & Média \\
\hline $\mathrm{P}_{2} \mathrm{O}_{5} 40 \%+\mathrm{K} 20 \%$ & $2,93^{(4)} \mathrm{b}$ & $2,73 b$ & $1,88 \mathrm{~b}$ & $2,93 b$ & $3,18 b$ & $3,26 a b$ & $2,15 b$ & $3,33 b$ & $2,53 \mathrm{ab}$ & $2,77 \mathrm{~b}$ \\
\hline $\mathrm{P}_{2} \mathrm{O}_{5} 40 \%+\mathrm{K} 20 \%$ & $2,50 b$ & $2,67 b$ & $2,23 b$ & $3,07 \mathrm{~b}$ & $3,03 \mathrm{~b}$ & $3,15 b$ & $2,35 \mathrm{ab}$ & $3,67 b$ & $1,63 b$ & $2,70 \mathrm{~b}$ \\
\hline Fosetyl-Al, $250 \mathrm{~g} \mathrm{hL}^{-1}$ & $2,55 b$ & $2,85 b$ & $2,60 b$ & $3,47 \mathrm{ab}$ & $3,37 \mathrm{ab}$ & $2,85 \mathrm{~b}$ & $2,23 \mathrm{ab}$ & $3,24 b$ & $1,78 \mathrm{~b}$ & $2,66 \mathrm{~b}$ \\
\hline Sem tratamento & $3,93 \mathrm{a}$ & $3,68 \mathrm{a}$ & $3,51 \mathrm{a}$ & $3,98 \mathrm{a}$ & $4,00 \mathrm{a}$ & $4,47 \mathrm{a}$ & $2,75 \mathrm{a}$ & $4,13 \mathrm{a}$ & $2,83 \mathrm{a}$ & $3,70 \mathrm{a}$ \\
\hline Média & 2,98 & 2,98 & 2,55 & 3,36 & 3,15 & 3,43 & 2,37 & 3,59 & 2,19 & - \\
\hline DMS* & 1,00 & 0,83 & $0,72-$ & 0,91 & 0,65 & 1,22 & 0,60 & 0,80 & 0,75 & 0,93 \\
\hline
\end{tabular}

* Diferença mínima significativa de Tukey $(\mathrm{P}<0,05)$.

(1) 1- 'NT Red'; 2- 'Golden'; 3- 'Baixinho de St. Amália'; 4- 'Grampola'; 5- 'Sunrise Solo'; 6- 'Cross Paris'; 7- 'Tailândia Verde'; 8- 'Tailândia Roxo'; 9- 'Tailândia Roxão'. (2) $\mathrm{P}_{2} \mathrm{O}_{5} 40 \%+\mathrm{K} 20 \%\left(150 \mathrm{~mL} \mathrm{hL}^{-1}\right)$. ${ }^{(3)} \mathrm{P}_{2} \mathrm{O}_{5} 40 \%+\mathrm{K} 20 \%\left(250 \mathrm{~mL} \mathrm{hL}^{-1}\right)$. (4) Os valores são relativos às médias de quatro repetições e de dez avaliações no tempo.

TABELA 2 - Efeito de fosfitos na severidade da varíola (Asperisporium caricae) em folhas de nove genótipos de mamoeiro, entre abril e julho de 2005, em condições de campo (Brasília-DF).

\begin{tabular}{|c|c|c|c|c|c|c|c|c|c|c|}
\hline \multirow{6}{*}{$\begin{array}{l}\text { Tratamento } \\
\mathrm{P}_{2} \mathrm{O}_{5} 40 \%+\mathrm{K} 20 \% \\
\mathrm{P}_{2} \mathrm{O}_{5} 40 \%+\mathrm{K} 20 \% \\
\text { Fosetyl-Al, } 250 \mathrm{~g} \mathrm{hL}^{-1} \\
\text { Sem tratamento }\end{array}$} & \multicolumn{10}{|c|}{ Genótipo - Severidade $(1-6)$ de varíola } \\
\hline & $1^{(1)}$ & 2 & 3 & 4 & 5 & 6 & 7 & 8 & 9 & Média \\
\hline & $4,10^{(4)} b$ & $3,78 b$ & $3,48 \mathrm{a}$ & $4,43 \mathrm{ab}$ & $4,14 \mathrm{ab}$ & $4,00 b$ & $3,93 \mathrm{ab}$ & 4,40 & $3,28 b$ & $3,95 \mathrm{~b}$ \\
\hline & $4,62 b$ & $3,83 b$ & $3,38 \mathrm{a}$ & $4,10 b$ & $3,68 b$ & $4,33 b$ & $3,70 b$ & 4,50 & $2,83 b$ & $3,88 \mathrm{~b}$ \\
\hline & $4,13 b$ & $3,88 b$ & $3,65 \mathrm{a}$ & $4,11 b$ & $3,90 b$ & $4,40 b$ & $3,53 b$ & 4,45 & $3,58 \mathrm{ab}$ & $3,96 \mathrm{~b}$ \\
\hline & $5,17 \mathrm{a}$ & $4,38 \mathrm{a}$ & $4,17 b$ & $4,78 \mathrm{a}$ & $4,33 \mathrm{a}$ & $5,26 \mathrm{a}$ & $4,25 \mathrm{a}$ & 4,59 & $3,85 \mathrm{a}$ & $4,53 \mathrm{a}$ \\
\hline Média & 4,50 & 3,96 & 3,67 & 4,35 & 4,01 & 4,50 & 3,85 & 4,48 & 3,38 & - \\
\hline DMS* & 0,49 & 0,50 & 0,52 & 0,67 & 0,43 & 0,86 & 0,55 & n.s. ${ }^{(5)}$ & 0,45 & 0,57 \\
\hline
\end{tabular}

* Diferença mínima significativa de Tukey $(\mathrm{P}<0,05)$.

(1) 1- 'NT Red'; 2- 'Golden'; 3- 'Baixinho de St. Amália'; 4- 'Grampola'; 5- 'Sunrise Solo'; 6- 'Cross Paris'; 7- 'Tailândia Verde'; 8- 'Tailândia Roxo'; 9- 'Tailândia Roxão'. (2) $\mathrm{P}_{2} \mathrm{O}_{5} 40 \%+\mathrm{K} 20 \%\left(150 \mathrm{~mL} \mathrm{hL}^{-1}\right)$. (3) $\mathrm{P}_{2} \mathrm{O}_{5} 40 \%+\mathrm{K} 20 \%\left(250 \mathrm{~mL} \mathrm{hL}^{-1}\right)$. (4) Os valores são relativos às médias de quatro repetições e de dez avaliações no tempo. ${ }^{(5)}$ n. s. = não-significativa.

TABELA 3 - Efeito de tratamentos com fosfitos na severidade da varíola (Asperisporium caricae) do mamoeiro ('Sunrise Solo'), entre março e abril de 2005, em casa de vegetação.

\begin{tabular}{ccc}
\hline Tratamento - princípio ativo $\left(\% \mathrm{~g} \mathrm{~g}^{-1}\right)$ & Dose de produto comercial & Severidade média (1-6)* \\
\hline $\mathrm{P}_{2} \mathrm{O}_{5} 40 \%+\mathrm{K} 20 \%$, & $150 \mathrm{~mL} \mathrm{hL}^{-1}$ & $2,73 \mathrm{~b}$ \\
$\mathrm{P}_{2} \mathrm{O}_{5} 40 \%+\mathrm{K} 20 \%$, & $250 \mathrm{~mL} \mathrm{hL}^{-1}$ & $2,66 \mathrm{~b}$ \\
$\mathrm{P}_{2} \mathrm{O}_{5} 30 \%+\mathrm{Ca} 6 \%$ & $400 \mathrm{~mL} \mathrm{hL}^{-1}$ & $2,91 \mathrm{~b}$ \\
$\mathrm{P}_{2} \mathrm{O}_{5} 20 \%+\mathrm{K} 20 \%$ & $200 \mathrm{~mL} \mathrm{hL}^{-1}$ & $2,57 \mathrm{~b}$ \\
$\mathrm{P}_{2} \mathrm{O}_{5} 30 \%+\mathrm{Ca} 7 \%$ & $300 \mathrm{~mL} \mathrm{hL}^{-1}$ & $2,46 \mathrm{~b}$ \\
$\mathrm{P}_{2} \mathrm{O}_{5} 30 \%+\mathrm{Mg} 4 \%$ & $300 \mathrm{~mL} \mathrm{hL}^{-1}$ & $2,91 \mathrm{~b}$ \\
$\mathrm{P}_{2} \mathrm{O}_{5} 40 \%+\mathrm{Mg} \mathrm{6} \%$ & $150 \mathrm{~mL} \mathrm{hL}^{-1}$ & $2,84 \mathrm{~b}$ \\
$\mathrm{P}_{2} \mathrm{O}_{5} 30 \%+\mathrm{K} 20 \%$ & $180 \mathrm{~mL} \mathrm{hL}^{-1}$ & $2,66 \mathrm{~b}$ \\
$\mathrm{P}_{2} \mathrm{O}_{5} 25 \%+\mathrm{Cu} 5 \%$ & $250 \mathrm{~mL} \mathrm{hL}^{-1}$ & $1,84 \mathrm{c}$ \\
$\mathrm{P}_{2} \mathrm{O}_{5} 20 \%+\mathrm{K} 20 \%$ & $200 \mathrm{~mL} \mathrm{hL}^{-1}$ & $2,80 \mathrm{~b}$ \\
Fosetyl-Al $80 \%$ & $250 \mathrm{~g} \mathrm{hL}^{-1}$ & $2,86 \mathrm{~b}$ \\
Testemunha sem aplicąão & - & $3,46 \mathrm{a}$ \\
\hline Diferença Mínima Significativa** & - & 0,54 \\
\hline
\end{tabular}

* Os valores são relativos às médias de cinco repetições e de sete avaliações no tempo.

** Teste de Tukey, $5 \%$.
TABELA 4 - Efeito de tratamentos com fosfitos na severidade da varíola (Asperisporium caricae) do mamoeiro ('Sunrise Solo'), entre maio e junho de 2005, em casa de vegetação.

\begin{tabular}{ccc}
\hline Tratamento - princípio ativo $\left(\% \mathrm{~g} \mathrm{~g}^{-1}\right)$ & Dose de produto comercial & Severidade média $(1-6)^{*}$ \\
\hline $\mathrm{P}_{2} \mathrm{O}_{5} 40 \%+\mathrm{K} 20 \%$ & $150 \mathrm{~mL} \mathrm{hL}^{-1}$ & $2,72 \mathrm{~b}$ \\
$\mathrm{P}_{2} \mathrm{O}_{5} 40 \%+\mathrm{K} 20 \%$ & $250 \mathrm{~mL} \mathrm{hL}^{-1}$ & $2,52 \mathrm{~b}$ \\
$\mathrm{P}_{2} \mathrm{O}_{5} 30 \%+\mathrm{Ca} 6 \%$ & $400 \mathrm{~mL} \mathrm{hL}^{-1}$ & $2,92 \mathrm{a}$ \\
$\mathrm{P}_{2} \mathrm{O}_{5} 20 \%+\mathrm{K} 20 \%$ & $200 \mathrm{~mL} \mathrm{hL}^{-1}$ & $2,66 \mathrm{~b}$ \\
$\mathrm{P}_{2} \mathrm{O}_{5} 40 \%+\mathrm{Mg} 6 \%$ & $150 \mathrm{~mL} \mathrm{hL}^{-1}$ & $2,54 \mathrm{~b}$ \\
$\mathrm{P}_{2} \mathrm{O}_{5} 30 \%+\mathrm{K} 20 \%$ & $180 \mathrm{~mL} \mathrm{hL}^{-1}$ & $2,72 \mathrm{~b}$ \\
$\mathrm{P}_{2} \mathrm{O}_{5} 20 \%+\mathrm{K} 20 \%$ & $200 \mathrm{~mL} \mathrm{hL}^{-1}$ & $2,70 \mathrm{~b}$ \\
Fosetyl-Al $80 \%$ & $250 \mathrm{~g} \mathrm{hL}^{-1}$ & $2,78 \mathrm{~b}$ \\
Testemunha sem aplicação & - & $3,14 \mathrm{a}$ \\
\hline Diferença Mínima Significativa** & & 0,36 \\
\hline
\end{tabular}

* Os valores são relativos às médias de cinco repetições e de cinco avaliações no tempo.

** Teste de Tukey, $5 \%$ 


\section{REFERÊNCIAS}

AGRIOS, G. N. Plant pathology. $4^{\text {th }}$ ed. San Diego: Academic Press, 1997.635p.

BOTEON, M. Desafios da fruticultura e o mercado de mamão. In: MARTINS, D.S. (Ed.). Papaya Brasil: mercado e inovações tecnológicas para o mamão. Vitória: Incaper, 2005. p. 15-21.

BRACKMAN, A.; GIEHL, R. F. H.; SESTARI, I.;STEFFENS, C. A. Fosfitos para o controle de podridões pós-colheita em maçãs Fuji durante o armazenamento refrigerado. Ciência Rural, Santa Maria, v.34, n. 34, p.1039-1042, 2004.

BRAPEX. Exportação. Associação Brasileira dos Exportadores de Papaya, 2007. Disponível em:< http://www.brapex.net/ index_1024.asp>.Acesso em:10 mar. 2008.

CELOTO, M. I. B.; PAPA, M. F. S.; CORREA, L. S. Efeito de fungicidas no controle da varíola (Asperisporium caricae) do mamoeiro. Summa Phytopathologica, Botucatu, v. 29, p. 87, 2003.

DIANESE, A. C.; BLUM, L. E. B.; DUTRA, J. B.; LOPES, L. F.; SENA, M. C.; FREITAS, L. F., YAMANISHI, O. K. Reação de genótipos de mamoeiro à varíola e à podridão-do-pé. Fitopatologia Brasileira, Lavras, v.32, n.5, p. 419-423. 2007.

HAGAN, A. Alternative treatments for turf disease. Grounds Maintenance, Lexington, v.40, n.3,p.10-12. 2005.

HOLDERNESS, M. Comparison of metalaxyl/cuprous oxide and potassium phosphonate as sprays and trunk injections for control of Phytophthora palmivora pod rot and canker of cocoa. Crop Protection, Amsterdan, v.11, p.141-147, 1992.

McDONALD, A. E.; GRANT, B. R.; PLAXTON, W. C. Phosphite (phosphorous acid): its relevance in the environment and agriculture and influence on plant phosphate starvation response. Journal of Plant Nutrition, Nova York, v.24, n.10, p.1505-1519, 2001.

PANICKER, S; GANGADHARAN, K. Controlling downy mildew of maize caused by Peronosclerospora sorghi by foliar sprays of phosphonic acid compounds. Crop Protection, Amsterdã, v.18, n.2, p.115-118, 1999.
REZENDE, J.A. M.; FANCELLI, M. I. 1997. Doenças do mamoeiro (Carica papaya). In: KIMATI, H., AMORIM, L., FILHO, A. B., CAMARGO; L. E. A.; REZENDE, J. A. M (Ed.). Manual de fitopatologia: doenças das plantas cultivadas. São Paulo: Agronômica Ceres, 1997. p.486-496.

RIBEIRO JÚNIOR, P. M.; RESENDE, M. L. V.; PEREIRA, R. B.; CAVALCANTI, F. R.; AMARAL, D. R.; PÁDUA, M. A. Fosfito de potássio na indução de resistência a Verticillium dahliae Kleb., em mudas de cacaueiro (Theobroma cacao L.). Ciência Agrotécnica, Lavras, v.30, n.4, p.629-636, 2006.

SANTANA, E. N. Controle químico das doenças fúngicas do mamoeiro em pré e pós-colheita pelo uso de fungicidas e ceras. 2004. Tese (Doutorado em Fitopatologia) - Faculdade de Agronomia, Universidade Federal de Viçosa, Viçosa, 2004.

SANTOS, M. C.; BARRETO, M. Estudo epidemiológico da varíola do mamoeiro em cultivares submetidos a tratamentos com fungicidas. Summa Phytopathologica, Botucatu, v.29, n.2, p.141-146, 2003.

SMILLIE, R.; GRANT, B. R.; GUEST, D. The mode of action of phosphite: evidence for both direct and indirect modes of action on three Phytophthora spp. in plants. Phytopathology, St. Paul, v.79, n.9, p.921-926, 1989.

TATAGIBA, J. S.; AGUILAR, M.A.G. Eficiência do Midas BR no controle da pinta preta (Asperisporium caricae) do mamoeiro (Carica papaya L.). In: MARTINS, D. S. (Ed.). Papaya Brasil: mercado e inovações tecnológicas para o mamão. Vitória: Incaper, 2005. p. 405-408.

VENTURA, J., A.; COSTA, H.; TATAGIBA, J. S. Manejo das doenças do mamoeiro. In: MARTINS, D. S.; COSTA, A. F. S. (Ed.). A cultura do mamoeiro: tecnologias de produção. Vitória: Incaper, 2003. p. 229-308.

ZAMBOLIM, L.; JUNQUEIRA, N. T. V.; ZAMBOLIM, E. M. Manejo integrado de doenças de fruteiras. In: CONGRESSO BRASILEIRO DE FRUTICULTURA, 19., 2006, Cabo Frio, RJ. Frutas do Brasil: saúde para o mundo.Cabo Frio: SBF/UENF/ UFRuralRJ, 2006. p. 19-35. 